\section{Vapor-Liquid Equilibrium Measurements of Polymeric Solutions - A Review}

\author{
lanatul Khoiroh
}

Department of Chemical \& Environmental Engineering, University of Nottingham Malaysia

\section{Ianatul.Khoiroh@nottingham.edu.my}

\section{Introduction}

The high demands for high-tech polymer materials lead to a tendency in the development of new methods for polymer in the separation processes. The efficient removal of unwanted components from the polymerization process, i.e., catalyst, monomer, and solvent molecules, is one of the main objectives during polymer finishing processing. The thermodynamic and phase equilibrium properties of polymer systems will affect how these processing steps can be carried out as well as an important factor to integrate production of polymers, proces and material selection (Folie et al., 1995). For this reason, knowledge of thermodynamic properties of polymer solutions is crucial for industry and research development (Wohlfarth, 1993). Moreover, the polymer processing often occurs in the dissolved state, and it is therefore, of practical importance in polymer process design to know the saturated pressures of the polymer solutions. Thus, the unwanted volatile materials such as solvents can be separated from polymer products in order to meet the environment, health and safety regulations. Such data are also served as an essential tool to describe the physical properties of polymeric materials and for further understanding to the nature of the intermolecular interactions. They will provide the necessary foundation for development of various thermodynamic models. A careful compilation of thermodynamics data are of practical importance for academic and industrial research. Whenever possible, the thermodynamic models should be based on reliable experimental data for a particular mixture at specific conditions of temperature, pressure and composition corresponding to those of interest. Unfortunately, such data are often scarcely available. As a result, an experimental technique needs to be improved for measuring more accurate data.
The nature of the molecule of the substance depends heavily on its physico-chemical properties. An accurate and reliable measurement of physical properties is therefore, essentially needed as a framework to gain a comprehensive understanding of the molecular behavior. Of these properties, saturated pressure is one of the most important thermophysical properties in the design of products, equipment, and processes in various chemical industries. In this review, various techniques that have been utilized in measuring the vapor-liquid equilibrium (VLE) for polymeric solutions are discussed.

Keywords: VLE, polymer, measurements, pressure.

Submitted: June $1^{\text {st }}, 2020$

Revised: june $15^{\text {th }}, 2020$

Accepted: September $28^{\text {th }}, 2020$

Published: September $29^{\text {th }}, 2020$

\section{Vapor-Liquid Equilibrium (VLE) Measurements}

Measurements of phase equilibrium data for mixtures composed of solvent and polymer are different than those for normal fluid systems, since diffusion of solvent into polymer is slow and vapor phase does not contain polymer as usual because polymer is non-volatile. The VLE measurements of polymer solutions, therefore, need to conducted by considering all these effects. Investigations on VLE of polymer solutions can be performed by employing various methods (Wohlfarth, 1993; Danner and High, 1993; Wen et al., 1992a; Wen et al., 1992b) including vapor pressure measurement, isopiestic method, gravimetric sorption, gas chromatofgraphy, and membrane osmometry which is only used marginally. The other two methods: light scattering and use of ultracentrifuge have each only been quoted in few references as methods to obtain solvent activity data in polymer solutions, but they are well known as methods for molecular weight determination. The details of the above-mentioned techniques will be summarized below.

\section{Vapor Pressure Measurements}

More than $80 \%$ data sets in DECHEMA for binary polymer solutions are obtained from determining the saturated vapor pressure of the solution. There are several ways to obtain the vapor pressures of polymer solutions. The obvious choice is to measure the absolute pressure, or as in the differential mode, to measure the difference between the vapor pressures of the pure solvent and the polymer solution. At lower concentrations, the availability of precise instruments are necessity, since the vapor pressure data will be close to those pure solvent with lowering amount of polymer. Hence, the measurements of absolute saturated needs to be performed carefully, longer duration, and

How to cite this article:

Khoiroh, I. (2020) Vapor-Liquid Equilibrium Measurements of Polymeric Solutions - A Review. Buletin Profesi Insinyur 3(2) 099-102 


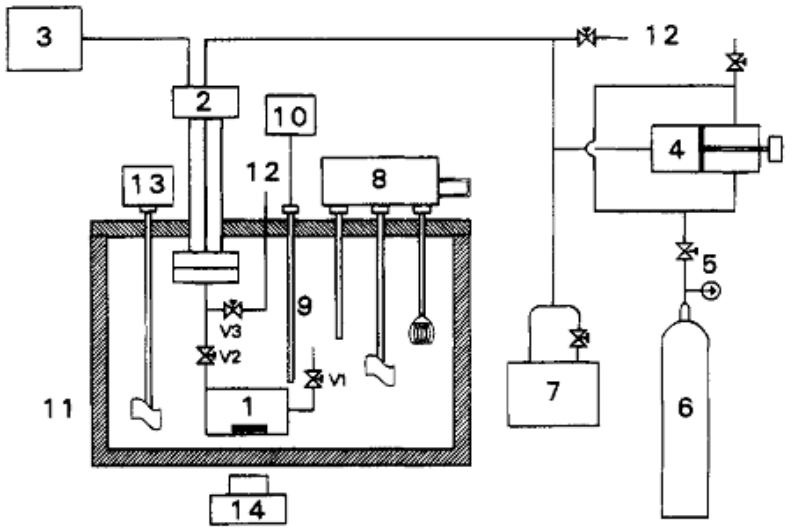

Figure 1. Schematic diagram of vapor pressure measurement (Esteve et al., 1995)

unsuitable for highly viscous polymer solutions (Wohlfarth, 1993).

As shown in Figure 1, the vapor pressure measurement method essentially consists of equilibrium cell, pressure and temperature sensors. Various researchers have reported the used and improved of this method to measure the VLE data of polymer or oligomer systems (Esteve et al., 1995; Herraiz et al., 1995; Haynes et al., 1998; Chaudhari et al., 1995; Xu et al., 1996; Lei et al., 2007; Khoiroh et al., 2000).

\section{Isopiestic Method}

Isopiestic measurement involves the determination of solvents' weight which are isothermally absorbed in certain amount of polymer while exposing the mixtures to a certain saturated pressure of solvent. In this technique, the polymer is assumed to be non-volatile. Mass detection can be determined by the change of length of a quartz spring (Gupta and Prausnitz, 1995; Lieu et al., 2000), a sensitive electronic microbalance, the equality of the solvent chemical potential through common standard solutions, or via a piezoelectric sorption detector called the quartz crystal microbalance (QCM) (Masuoka et al., 1984). The latter technique is illustrated in Figure 2 as an example. The principle of the method is based on the linear relationship between frequency shift and mass shift at the surface of the crystal as first described by Sauerbrey (Mikkilineni et al., 1995). One of the advantages of employing this method is the high sensitivity of the VLE data obtained. This is because QCM utilized small fractions of polymer spread in a thin film and therefore, the phase equilibrium can be obtained at fasted speed (Wong et al., 1997). The major disadvantage of this method is that it cannot be used at high solvent vapor pressures and therefore, QCM technique can be applied to only small range of polymer concentrations (French and Koplos, 1999; Price et al., 2006; Wibawa et al., 2002).

\section{Inverse Gas Chromatography}

Figure 3 illustrates the schematic diagram of an inverse gas chromatography (IGC) method. The apparatus consists of a short column packed with an inactive support material, where the polymer is dispersed as a thin film on the surface. Inert gas is used as a carrier with uniform flowrate through the column. A suitable detector is installed to detect the small volume of solvent which is injected at one end of the column. The experimental setup is in many ways the same as the infinite dilution studies, only now, the carrier gas contains a constant concentration level of the solvent.

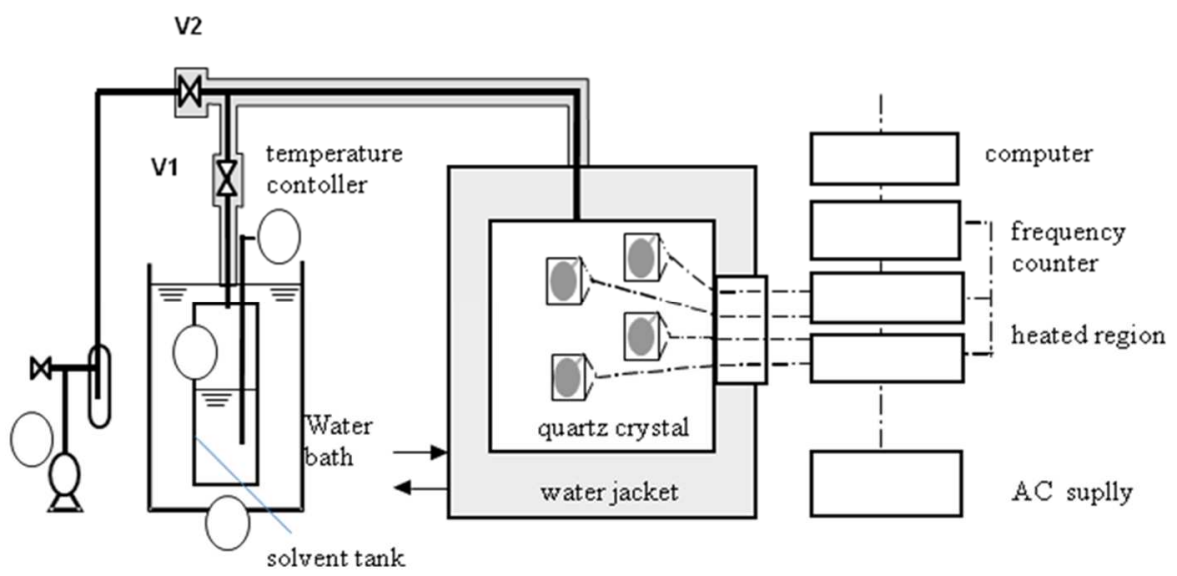

Figure 2. Schematic diagram of quartz crystal microbalance (Price et al., 2006). 


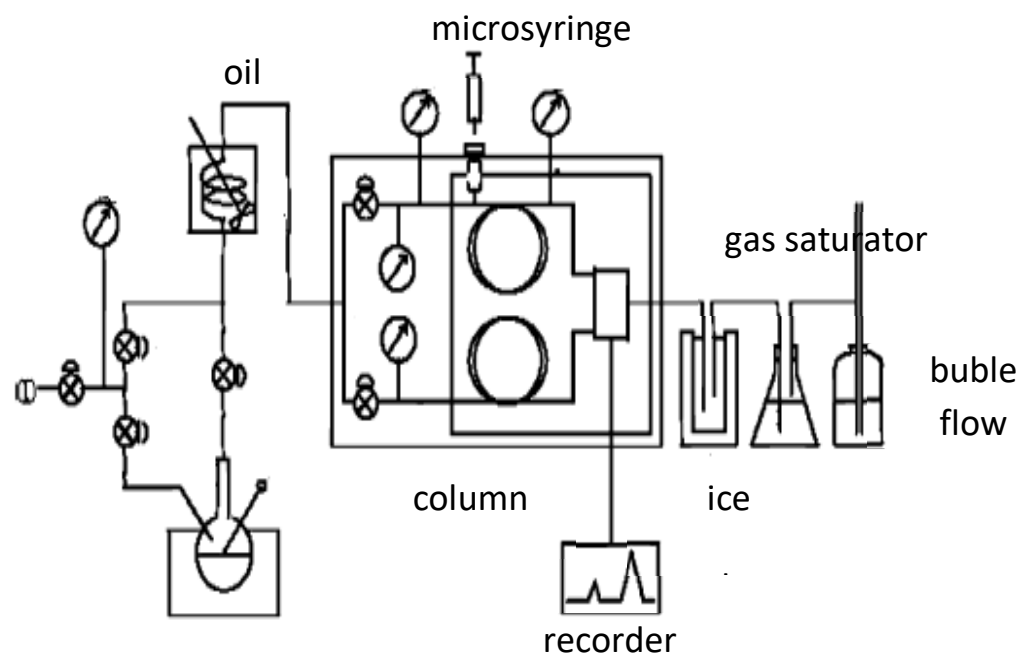

Figure 3. Schematic diagram of gas chromatography apparatus (Tochigi et al., 2005)

Solvent concentration in the polymer solution is varied by changing the level of solvent vapor in the carrier gas. The peak area and the retention time of the studied mixtures are measured by varying the flow rates at various temperatures and varying temperatures at a certain flow rate setting. IGC method is a fast and reliable technique for measurement of the activity coefficient and diffusivity for small molecules in polymers, particularly in the highly polymer concentrated region. In the process of application, this method has been modified and developed for special purpose of research, for example, in determination of activity of solute in polymer solutions at essentially zero solute concentration (Tochigi et al., 2005; Bercea et al., 2009).

\section{Membrane Osmometry}

The principle of membrane osmometry is simple (Billmayer, 1971). A polymer solution is separated from the pure solvent by semipermeable membrane as indicated in Figure 4, through which only solvent molecules can penetrate. To compensate for the difference between the solvent activity in the pure state and in the polymer solution, the solvent molecule will diffuse from the pure solvent into the polymer solution. This diffusion will result in difference in the liquid level of the two sections (measured by connected capillary tubes).

After equilibrium has been reached the corresponding hydrostatic pressure will be equal to the osmotic pressure, $\pi$, which again can be related to the solvent activity, a1, by the following equation:

$$
\ln a_{1}=-\frac{\pi V_{1}}{R T}
$$

where $V_{1}$ is the molar volume of the solvent.

\section{Conclusion}

The separations of the fluid mixtures into their chemical constituents in chemical process industries are designed based on attaining phase equilibria data. As a consequence, the development of perspective separation technologies requires quantitative saturated pressure data for mixtures of interest. It is then clear why the measurements of saturated pressures have been the subject of considerable effort for various compounds and mixtures such as polymeric solutions.

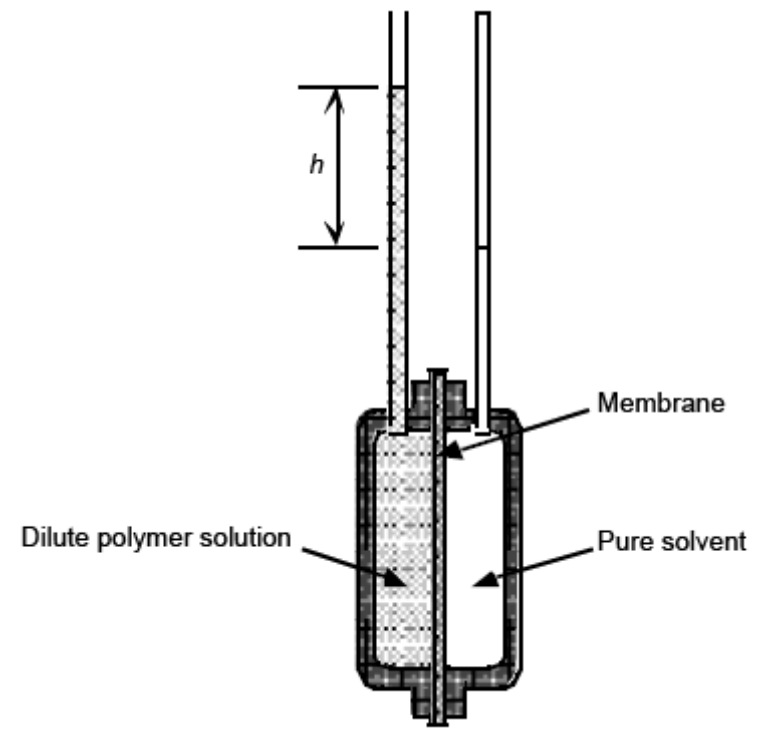

Figure 4. Schematic diagram of simple membrane osmometry method (Fried, 1995).

\section{Acknowledgement}

The author would like to thank Prof. Iryanti Fatyasari Nata and Prof. Chairul Irawan for valuable discussions.

\section{References}

Bercea, M.; Eckelt, J.; Wolf, B. A., (2009) Vapor Pressures of Polymer Solutions and the Modeling of Their Composition Dependence. Ind. Eng. Chem. Res., 48, (9), 4603-4606. 
Billmayer, F. W. (1971) Textbook of Polymer Science. Wiley: New York.

Chaudhari, S. K.; Patil, K. R.; Allepús, J.; Coronas, A., (1995) Measurement of the Vapor Pressure of 2,2,2Trifluoroethanol and Tetraethylene Glycol Dimethyl Ether by Static Method. Fluid Phase Equilib. 108, (1-2), 159-165.

Danner, R. P.; High, M. S. (1993) Handbook of Polymer Solution Thermodynamics. Wiley: New York.

Esteve, X.; Chaudhari, S. K.; Coronas, A. (1995) Vapor-Liquid Equilibria for Methanol + Tetraethylene Glycol Dimethyl Ether. J. Chem. Eng. Data, 40, (6), 1252-1256.

Folie, B.; Radosz, M. (1995) Phase Equilibria in HighPressure Polyethylene Technology. Ind. Eng. Chem. Res., 34, (5), 1501-1516.

French, R. N.; Koplos, G. J. (1999) Activity Coefficients of Solvents in Elastomers Measured with a Quartz Crystal Microbalance. Fluid Phase Equilib., 158-160, (0), 879892.

Fried, J. R. (1995) Polymer Science and Technology. Prentice Hall: New Jersey.

Gupta, R. B.; Prausnitz, J. M. (1995), Vapor-Liquid Equilibria of Copolymer + Solvent and Homopolymer + Solvent Binaries: New Experimental Data and Their Correlation. J. Chem. Eng. Data 1995, 40, (4), 784-791.

Haynes, C. A.; Beynon, R. A.; King, R. S.; Blanch, H. W. Prausnitz, J. M., (1989) Thermodynamic Properties of Aqueous Polymer Solutions: Poly(ethylene Glycol)/Dextran. J. Phys. Chem. 93, (14), 5612-5617.

Herraiz, J.; Shen, S.; Coronas, A. (1998) Vapor-Liquid Equilibria for Methanol + Poly(ethylene glycol) 250 Dimethyl Ether. J. Chem. Eng. Data 1998, 43, (2), 191195.

Khoiroh, I.; Lee, M.-J (2011)., Isothermal Vapor-Liquid Equilibrium for Binary Mixtures of Polyoxyethylene 4Octylphenyl Ether with Methanol, Ethanol, or Propan-2ol. J. Chem. Eng. Data 2011, 56, (4), 1178-1184.

Lei, H.; Wilding, W. V.; Pitt, W. G. (2007) Measurement of Activities of Toluene and Trichloroethylene in Polyisobutylene. J. Chem. Eng. Data 2007, 52, (6), 2233 2236.

Lieu, J. G.; Prausnitz, J. M.; Gauthier, M., (2000) VaporLiquid Equilibria for Binary Solutions of Arborescent and Linear Polystyrenes. Polymer 2000, 41, (1), 219-224.
Masuoka, H.; Murashige, N.; Yorizane, M. (1984) Measurement of Solubility of Organic Solvents in Polyisobutylene Using the Piezoelectric-Quartz Sorption Method. Fluid Phase Equilib. 18, (2), 155-169.

Mikkilineni, S. P. V. N.; Tree, D. A.; High, M. S., Thermophysical Properties of Penetrants in Polymers via a Piezoelectric Quartz Crystal Microbalance. J. Chem. Eng. Data 1995, 40, (4), 750-755.

Price, G. J.; Haddon, D. A.; Bainbridge, A.; Buley, J. M. (2006) Vapour Sorption Studies of Polymer-Solution Thermodynamics Using a Piezoelectric Quartz Crystal Microbalance. Polym. Int. 55, (7), 816-824.

Tochigi, K.; Kurita, S.; Okitsu, Y.; Kurihara, K.; Ochi, K., (2005)Measurement and Prediction of Activity Coefficients of Solvents in Polymer Solutions Using Gas Chromatography and a Cubic-Perturbed Equation of State with Group Contribution. Fluid Phase Equilib, 228229, (0), 527-533.

Wen, H.; Elbro, H. S.; Alessi, P. (1992a) Polymer Solution Data Collection Part 1. DECHEMA Frankfurt.

Wen, H.; Elbro, H. S.; Alessi, P. (1992b) Polymer Solution Data Collection Part $2+3$. DECHEMA Frankfurt.

Wibawa, G.; Takahashi, M.; Sato, Y.; Takishima, S.; Masuoka, H. (2002) Solubility of Seven Nonpolar Organic Solvents in Four Polymers Using the Piezoelectric-Quartz Sorption Method. J. Chem. Eng. Data 2002, 47 (3), 518524.

Wohlfarth, C. (1993) Calculation of Phase Equilibria in Random Copolymer Systems. Makromol. Theory Simul. 1993, 2, (5), 605-635.

Wohlfarth, C. (1994) Vapour-Liquid Equilibrium Data of Binary Polymer Solutions: Vapour Pressures, HenryConstants and Segment-Molar Excess Gibbs Free Energies. Elsevier Science B. V.: Amsterdam.

Wong, H. C.; Campbell, S. W.; Bhethanabotla, V. R. (1997) Sorption of Benzene, Toluene and Chloroform by Poly(styrene) at $298.15 \mathrm{~K}$ and $323.15 \mathrm{~K}$ Using a Quartz Crystal Balance. Fluid Phase Equilib. 1997, 139, (1-2), 371-389.

Xu, R.; Léonard, J.; Bui, V. T. (1996) Vapor Pressure for Mixtures of Methylene Ester Oligomers with p-Dioxane and Chloroform. J. Chem. Eng. Data 1996, 41, (4), 681684. 